\title{
Plasticity during vestibular compensation: the role of saccades
}

\section{Hamish Gavin MacDougall and lan S. Curthoys*}

Vestibular Research Laboratory, School of Psychology, The University of Sydney, Sydney, NSW, Australia

Edited by:

Pierre-Paul Vidal, Universite Rene

Descartes/CNRS, France

\section{Reviewed by:}

Ted Raphan, Brooklyn College of CUNY, USA

Seong-Hae Jeong, Chungnam

National University Hospital,

South Korea

\section{*Correspondence:}

Ian S. Curthoys, Vestibular Research Laboratory, School of Psychology, The University of Sydney, A18, Sydney, NSW, Australia.

e-mail: ianc@psych.usyd.edu.au
This paper is focused on one major aspect of compensation: the recent measures of saccadic responses to high acceleration head turns during human vestibular compensation and their possible implications for recovery after unilateral vestibular loss (UVL). New measurement techniques have provided additional insights into how patients recover after UVL and have given clues for vestibular rehabilitation. Prior to this it has not been possible to quantify the level of function of all the peripheral vestibular sense organs. Now it is. By using vestibular-evoked myogenic potentials to measure utricular and saccular function and by new video head impulse testing to measure semicircular canal function to natural values of head accelerations. With these new video procedures it is now possible to measure both slow phase eye velocity and also saccades during head movements with natural values of angular acceleration. The present evidence is that after UVL there is little or no restoration/compensation of slow phase eye velocity responses to natural head accelerations. It is doubtful as to whether the modest changes in slow phase eye velocity to small angular accelerations are functionally effective during compensation. On the other hand it is now clear that saccades can play a very important role in helping patients compensate and return to a normal lifestyle. Preliminary evidence suggests that different patterns of saccadic response may predict how well patients recover. Furthermore it may be possible to train patients to produce more effective saccadic patterns in the first days after their unilateral loss and possibly improve their compensation process. Some patients do learn new strategies, new behaviors, to conceal their inadequate vestibulo-ocular response but when those strategies are prevented from operating by using passive, unpredictable, high acceleration natural head movements, as in the head impulse test, the vestibular loss can be demonstrated. It is those very strategies which the tests exclude, which may be the cause of their successful compensation.

Keywords: labyrinth, otolith, bone conduction, oVEMP, utricular, saccular, semicircular canal

\section{OVERVIEW}

The vestibular receptors of the inner ears detect head movements and generate corrective eye movements and postural corrections which maintain a stable visual image and a stable person during head movements. A simple head movement produces an imbalance of neural activity between the two vestibular nuclei which results in these responses. The field of vestibular function and vestibular compensation is huge and there are many reviews of this very large area in detail (e.g., Curthoys and Halmagyi, 1995, 1999; Halmagyi et al., 2010). This review will focus on the changes in oculomotor behavior during compensation, in particular the role of saccades. Nevertheless we start with a brief general introduction which provides the basis for understanding the complex multidimensional control of eye movements.

Abbreviations: ACS, air-conducted sound; BCV, bone-conducted vibration; $\mathrm{COR}$, cervico-ocular response; cVEMPs, cervical vestibular-evoked myogenic potentials; $\mathrm{Fz}$, the location on the forehead in the midline at the hairline; HIT, head impulse test; oVEMPs, ocular vestibular-evoked myogenic potentials; SCM, sternocleidomastoid muscle; UVL, unilateral vestibular loss; vHIT, video head impulse test; VOR, vestibulo-ocular response.
The two vestibular nuclei in the brainstem are centers for integrating sensory input from many sources and generating multiple outputs. They receive neural input from the vestibular sensor organs in the inner ear, from proprioceptors in neck muscles, from distant levels of the spinal cord, from the visual system, from nuclei concerned with autonomic function as well as from the reticular formation and from the cerebellum. The output of these vestibular nuclei are almost as diverse - controlling eye movements, posture, autonomic responses, and generating sensations. Most importantly the two vestibular nuclei are interconnected by functionally inhibitory connections, called commissural connections.

So is it a simple "input-output" system? No. The operation of this whole system depends on how the vestibular sensory input was generated and what the person's goals are. If the sensory input is due to "passive," involuntary head movements then many corrective reflexes are initiated. If exactly the same vestibular sensory input is generated by the subject actively generating the same head movement, these reflexes are suppressed. For example a skater or an acrobat during an active spin suppresses compensatory responses. That suppression could take place by cerebellar input 
suppressing the transmission of information from the vestibular sensors to the various outputs.

At the simplest level, movements generate an imbalance of neural activity between the vestibular nuclei but unilateral disease or loss (which we will term UVL - for unilateral vestibular loss) also generates a very similar imbalance by silencing the neural input from one labyrinth. In both cases - the movement and the UVL - the responses and the sensations are similar - the person perceives themselves (or the room) as rotating (a sensation called vertigo), there are rhythmic eye movements (called nystagmus) and there are corrective postural responses. Put this way one sees that the symptoms of UVL consist of appropriate corrective responses for a large head rotation.

\section{MAJOR ACUTE SYMPTOMS OF UVL}

The most basic distinction is between static and dynamic symptoms and the following table lists just the most obvious of these symptoms occurring immediately after UVL.

\begin{tabular}{ll}
\hline $\begin{array}{l}\text { Static symptoms } \\
\text { (present with the } \\
\text { head stationary) }\end{array}$ & $\begin{array}{l}\text { Dynamic symptoms } \\
\text { (occurring when the individual } \\
\text { makes head movements) }\end{array}$ \\
\hline $\begin{array}{l}\text { Rhythmic eye movements - } \\
\text { nystagmus - even in light } \\
\text { with quick phases directed }\end{array}$ & $\begin{array}{l}\text { Reduced vestibulo-ocular gain for head } \\
\text { turns toward the affected side ("ipsile- } \\
\text { away from the affected side }\end{array}$ \\
$\begin{array}{l}\text { vertigo - the sensation that head turns) } \\
\text { the person is turning or that } \\
\text { the world is turning }\end{array}$ & $\begin{array}{l}\text { The world appears to move or jump } \\
\text { Postural unsteadiness - the }\end{array}$ \\
$\begin{array}{l}\text { patient tends to fall toward } \\
\text { called oscillopsia = "bouncing vision" }\end{array}$ \\
with eyes closed
\end{tabular}

Most real-life head movements are usually of short duration and at the end of the movement the neural activity in the vestibular nuclei return to their balanced state. But after UVL the imbalance in neural activity persists for many hours or days. In most patients many of these symptoms diminish within days or weeks and most patients return to their normal lifestyle. The term that is used to describe that overall recovery is "vestibular compensation." The main focus in this area has been on understanding the changes in the central nervous system which underlie this compensation.

In perhaps $20-30 \%$ or so of UVL patients - so-called "poorly compensated patients" - this recovery does not happen: they continue to be troubled by some or all of these symptoms. Probably the most difficult question of all in this area is - why don't these patients compensate adequately? Two considerations:

- Some of these poorly compensated patients may have had inadequate vestibular function on the "healthy" side or even central (e.g., cerebellar) deficits before the UVL procedure.

- "Recovery" has a large subjective component and it is probable that some of these patients had expected a much better outcome. What defines good vestibular compensation for human patients? It seems to be a return to their normal lifestyle, untroubled by smeared visual input during head movements, or the subjective sensations of bouncing vision (called oscillopsia).

Detailed comparison of the vestibular function of such patients so far has not been able to identify any clear differences post-UVL between well- and poorly compensated patients.

Vestibular compensation appears to be a simple general recovery of vestibular function with the passage of time. Closer examination shows a complex story which is now being clarified thanks to new vestibular tests which allow objective measures of the functional status of all vestibular sense organs. These tests are fast simple and safe and can be carried out even during severe attacks of vertigo immediately after UVL and they allow for repeated testing during the vestibular compensation process. First we need to consider how peripheral vestibular function is measured and we will consider semicircular canals and otoliths separately. We then consider new data from applying these measures to patients with UVL.

\section{MEASUREMENT OF SEMICIRCULAR CANAL FUNCTION}

Measurement of the vestibular contribution to the corrective eye movement response to head rotation is a major issue. Initially people used smooth sinusoidal head rotations at low frequencies and low accelerations, measuring the smooth slow phase eye movement response to these predictable head movement stimuli and inferring vestibular function from those measures. The standard measure of peripheral vestibular function has been the gain of the vestibulo-ocular response (VOR) which is the ratio of the size of the slow phase corrective eye movement response to the size of the passive head movement stimulus. So perfect gain is a gain of -1.0 , i.e., the eye movement is exactly equal and opposite to head movement and thus the eye movement exactly corrects for the head movement, with the result that the retinal image remains stable during the head movement.

We showed that such a measurement procedure is flawed. We reported a patient with definite absence of vestibular input bilaterally due to surgical removal of both vestibular nerves, who could still generate smooth eye slow phase eye movements to such predictable, low frequency, low acceleration rotational stimuli (Halmagyi and Curthoys, 1987). We reasoned that if a person without any vestibular function can generate the response, the measure is not a specific measure of vestibular function. So we concluded that the smooth slow phase eye movement response to low frequency, low acceleration, predictable sinusoidal rotations stimuli is a flawed indicator of specifically vestibular function.

When a different stimulus was used - a passive, unpredictable, head rotation with high (natural) accelerations (a "head impulse" in our terms), and the patient with bilaterally absent vestibular function was instructed to maintain gaze on a fixed target on the wall during the head impulse, the outcome was totally different. The objective measures of the eye movement response showed the bilateral patient did not generate an eye movement response during the first $70 \mathrm{~ms}$ of the head rotation, whereas healthy subjects did generate a response which closely matched and corrected for the head movement stimulus. So we concluded that this head impulse test (HIT) is a specific indicator of vestibular function. 
At that time (the late 1980s) measurement of the eye movement response to these stimuli required recording of the eye movement using scleral search coils which is an invasive, complex, and fearsomely expensive procedure totally unsuited to routine clinical use. However in testing these patients we noted a characteristic behavioral sign which could be used clinically. It is that during a head turn to their affected side, the patient's eyes moved with the head so that at the end of the head turn the patient made a saccade to regain fixation on the target as they had been instructed to do. That corrective saccade at the end of the head turn was the tell-tale sign of reduced or absent unilateral vestibular function on the side to which the head was turned. We now call it an "overt" saccade and it is a sign of loss of semicircular canal function. It occurs because the inadequate eye movement response during the ipsilesional head rotation means that during the head rotation the patient's eyes are dragged, with the head, off the target, and so at the end of the head rotation the patient makes a saccade which takes the patient's gaze back to the target. It is that overt corrective saccade which the clinician can detect by visual observation and which is the tell-tale sign of an inadequate VOR.

A simple version of this HIT can be conducted anywhere. This test consists of the clinician facing the patient and holding the patient's head at arm's length and then turning the patient's head in an abrupt unpredictable horizontal head rotation of about $20^{\circ}$ in less than $1 \mathrm{~s}$ while the patient is asked to stare at the tip of the clinician's nose and not blink. Although it is small and very brief, this kind of abrupt, passive head rotation has a peak angular velocity of around $200 \mathrm{deg} / \mathrm{s}$ and a peak angular acceleration of 2500$3000 \mathrm{deg} / \mathrm{s} / \mathrm{s}$. In healthy subjects this head rotation results in a short-latency (about $10 \mathrm{~ms}$ ) smooth compensatory eye movement so that the gaze remains fixed on the clinician's nose irrespective of whether the rotation is to the left or right. This means that in normal subjects VOR gain is close to -1.0 . For a patient after a UVL, the result is very different. If the patient's head is rotated to their affected side (an "ipsilesional" rotation) there is a marked reduction in VOR gain whereas for head rotations to the healthy side ("contralesional" rotations) the VOR gain is only modestly reduced.

Why is HIT such a specific test of semicircular canal function? Because the response is too early for other eye movement control mechanisms to be able to generate the slow phase eye velocity to correct for the head rotation. In the bilateral patient, their influence only appeared at about $70 \mathrm{~ms}$ after onset of head rotation.

This clinical sign is the overt saccade at the end of the head turn to the affected side and it relies on the clinician being able to detect the corrective saccade, and obviously an objective measure of the response is to be preferred over subjective visual observation. In some patients it is extremely difficult to detect an overt saccade at all. Scleral search coil measures have only been conducted on a relatively small number of patients because of the great difficulty and expense. Recently we have developed a small, light-weight, fast, head-mounted video camera system which allows accurate measures of eye movement during the head rotation. This new video procedure (called the video head impulse test, vHIT) allowed us to measure the VOR (and the saccades) in a very large number of patients. The accuracy, reliability, sensitivity, and specificity of vHIT were verified by comparing exactly simultaneous measures of eye movements with vHIT and search coils (MacDougall et al., 2009). After UVL and we have discovered response patterns which are very widespread among patients, which we had not appreciated with search coil recordings because of the relatively small number of patients tested. In particular we now contend that the corrective saccade is of great importance in understanding plasticity during recovery from UVL.

\section{MEASUREMENT OF OTOLITH FUNCTION}

Until about 20 years ago, clinical testing of otolith function was virtually impossible in standard clinics. To deliver the linear acceleration stimulus, patients were moved on sleds or centrifuges or tilt chairs or rotated off vertical axis. Most of these methods are potentially dangerous and most are totally clinically impractical. The physiological evidence that some primary otolith afferent neurons in guinea pigs have a very sensitive response to sound and vibration has underwritten a whole new approach to clinical testing of otolith function (see Curthoys and Vulovic, 2011 for a recent review). It is now clear that a small group of otolith afferents, otolith afferents with an irregular resting discharge, have a low threshold and a very sensitive response to bone-conducted vibration (BCV) and air-conducted sound (ACS; Murofushi et al., 1995; Curthoys et al., 2006; Curthoys and Vulovic, 2011) $500 \mathrm{~Hz}$ ACS or BCV activates a high proportion of these special very sensitive otolith irregular neurons from the utricular and saccular maculae and most are activated with great sensitivity at very low stimulus values - close to ABR threshold. BCV and ACS do activate afferents from other sensory regions, but usually in a totally different fashion - very high intensities are needed and even then the response is very modest. In other words the vestibular sense organs which are most sensitive to ACS and BCV are the utricular and saccular maculae. Clearly in response to any clinical tests stimulus it is these low threshold, high sensitivity neurons which will be activated at low intensities and presumably be responsible for the major part of any response. With ACS the neural responses are similar to those evoked by $\mathrm{BCV}$, although the stimulus intensities required are very high (60-80 dB above ABR threshold around 120-130 dB SPL).

Using this selective response of otolithic afferents to ACS and BCV has justified the use of ACS and BCV to index dynamic otolith function in the clinic. For clinical testing the BCV is usually delivered to the midline of the forehead at the hairline (a skull location called $\mathrm{Fz}$ ). The stimulus is either a light tap to $\mathrm{Fz}$ with a tendon hammer or a brief burst of modest vibration (about the intensity delivered by a body massager or an electric toothbrush) delivered by a Bruel and Kjaer minishaker 4810 (Curthoys, 2010).

\section{CERVICAL VESTIBULAR-EVOKED MYOGENIC POTENTIAL}

Sound-evoked saccular neurons project to and synapse on neurons in the ipsilateral vestibular nuclei and inhibitory neurons in the vestibular nuclei project ipsilaterally to spinal motoneurons and inhibit them (Uchino et al., 2005). In healthy subjects short tone bursts of $500 \mathrm{~Hz}$ of either high intensity ACS or moderate BCV result in a stimulus-locked short-latency inhibitory myogenic potential recorded by electrodes over tensed sternocleidomastoid muscles (SCM). This is the cervical vestibular-evoked myogenic potential (cVEMP) and it consists of an initial positive (inhibitory) potential ( $\mathrm{p} 13$ ) followed by a negative potential (n23; 
Colebatch and Halmagyi, 1992; Colebatch et al., 1994; Rosengren et al., 2010). The cVEMP p13-n23 is an uncrossed, descending, inhibitory, sacculocollic response. In patients with complete UVL following vestibular schwannoma removal there is a reduced or absent cVEMP p13-n23 from the ipsilateral SCM in response to Fz BCV or ACS stimulation of the affected ear. The absolute value of the cVEMP potential depends on many variables such as neck muscle tension and electrode placement. The diagnostically valuable information is the relative amplitude of the potentials over each SCM in response to symmetrical stimulation of both ears.

\section{OCULAR VESTIBULAR-EVOKED MYOGENIC POTENTIAL}

In response to BCV from a tendon hammer or a minishaker, surface EMG electrodes beneath the eyes record a stimulus-locked ocular vestibular-evoked myogenic potential (oVEMP) and the first negative (excitatory) component of which, at a latency of about $10 \mathrm{~ms}$, is called oVEMP $\mathrm{n} 10$. This component probably indicates primarily the myogenic potential of the inferior oblique and inferior rectus eye muscles, since the size of oVEMP n10 increases as the subject looks up and brings these muscles close to the electrodes. But when any healthy subject is given the Fz BCV stimulus which stimulates both labyrinths about equally it is found that the amplitude of the oVEMP n10 is similar beneath both eyes. The oVEMP is a crossed, excitatory, ascending, utriculo-ocular response (Rosengren et al., 2005; Iwasaki et al., 2007; Curthoys, 2010).

Stimulation of one ear by ACS also elicits oVEMP $\mathrm{n} 10$ responses beneath the contralateral eye, however the sound intensities needed are high and the n 10 potentials to ACS are typically small. In some subjects ACS also produces small potentials beneath the ipsilateral eye, but it is the amplitude of the contralateral oVEMP n10 which is of diagnostic value. ACS has the major disadvantage that conductive hearing loss renders the test results meaningless.

These new vHIT and VEMP tests allow measurement of the function of all 10 vestibular sensory regions using oVEMPs and cVEMPs (Curthoys, 2010) so that it is possible to identify the exact level of patient vestibular function even during acute vertigo after UVL. We now consider what these measures show after UVL.

\section{THE UVL}

In animal studies of vestibular compensation healthy animals are given a surgical complete unilateral loss. The surgical removal of the vestibular nerve is identified as the marker for commencement of compensation and that is reasonable in animal studies. Human patients undergo similar surgical complete removal of the vestibular nerve which deprives the person of all vestibular input from one labyrinth. However with human patients one has to consider the reasons for the surgery and those reasons are crucial in understanding the course of vestibular compensation. In most cases the animal and human situations are totally different. Animals are perfectly healthy prior to the UVL, whereas with humans the surgery takes place to remove a tumor which has been growing slowly, possibly for years, and so progressively depriving the individual of vestibular sensory input and allowing progressive compensation to take place before the surgery. Similarly patients who receive unilateral intratympanic injections of gentamicin receive that procedure because of vestibular dysfunction. So after schwannoma removal or intratympanic gentamicin, the UVL symptoms of such patients can be very minor and disappear very rapidly. This is a misleadingly fast "compensation."

The closest analog to the laboratory animal UVL are healthy individuals who suddenly lose all vestibular input on one side due to a total unilateral vestibular neuritis. Just how well do such patients recover? What should be categorized as vestibular compensation? Simple recovery of vestibular-related responses? One has to be careful in answering this question since after vestibular neuritis, peripheral vestibular function can return, so such patients are not really examples of vestibular compensation but vestibular "restoration" (Halmagyi et al., 2010). One great benefit of the new specific tests described above is that they allow the clinician to identify such restoration of peripheral vestibular function. So consider the results in Figure 1; Manzari et al., 2011, with permission).

\section{VESTIBULAR RESTORATION}

Figure 1 shows the results of vestibular tests from a patient diagnosed with superior vestibular neuritis. The patient's vestibular function was measured at the height of an acute attack of vertigo due to unilateral vestibular neuritis, and then again 3 months later, without any special medication in the interim. The results show that during the head turn to the affected ear (an ipsilesional head turn) at attack, there is a major drop in VOR gain for passive, unpredictable, high acceleration rotations. But at testing 3 months later the VOR for these ipsilesional rotations had fully returned. Similarly the test of utricular function (the oVEMP) at the acute attack shows an absent oVEMP n10 contralateral to the lesion indicating that the utricular function on the affected side was not present at the acute phase, but at 3 months later utricular function had fully returned. (In this patient, the saccular function was not affected by the neuritis - it was present at the acute phase and also at recovery, probably because the neuritis was confined to the superior division of the vestibular nerve).

Without the results of the tests of peripheral vestibular function and the demonstrated recovery of vestibular function after the neuritis, this would be classed as case of excellent vestibular compensation. It is not. The objective measures of peripheral vestibular function show the compensation is due to actual restoration of peripheral vestibular sensory function. The new measures have shown this example is misleading - peripheral function has returned whereas vestibular compensation is usually concerned with recovery associated with central changes.

In other VN patients this restoration of peripheral vestibular sensory function does not happen. And in our view it is just this small subgroup of vestibular neuritis patients who are equivalent to the usual animal model of compensation and it is this response of this small group of patients which should be studied intensively. It is wise to be cautious about the source of the evidence.

\section{RECOVERY OF DYNAMIC VOR SLOW PHASE EYE VELOCITY}

Early studies of dynamic VOR recovery indicated that there was considerable recovery of dynamic VOR. But for practical reasons those studies were restricted to measuring the slow phase eye velocity response to low frequency, low acceleration sine-wave rotations (with accelerations of the order of tens of degrees/second/second). 
Acute - 29 December 2010

Recovered - 21 March 2011
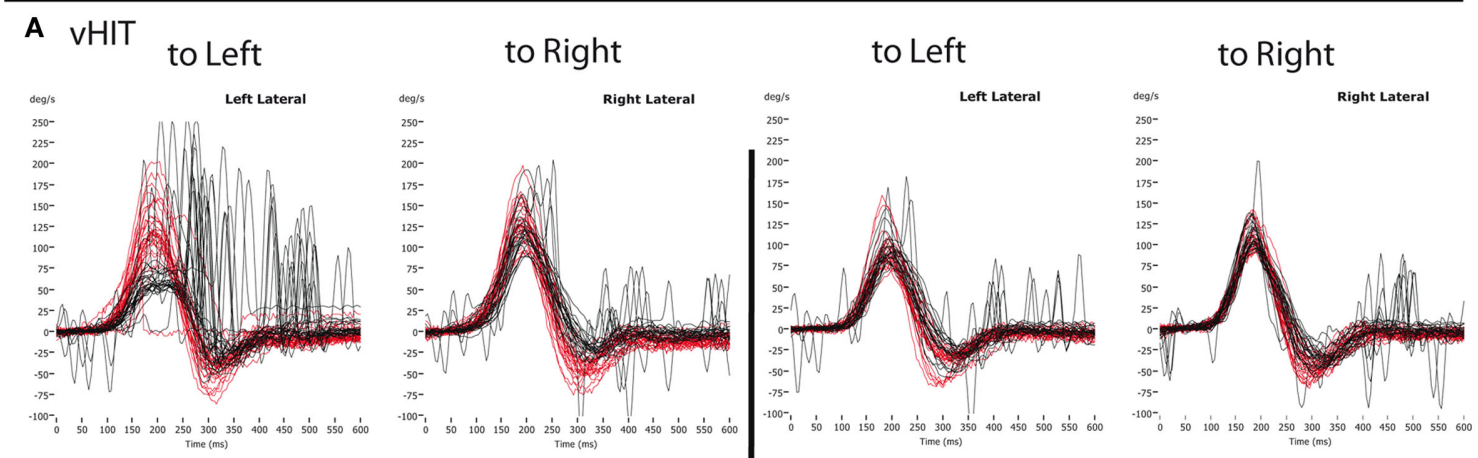

B Horizontal
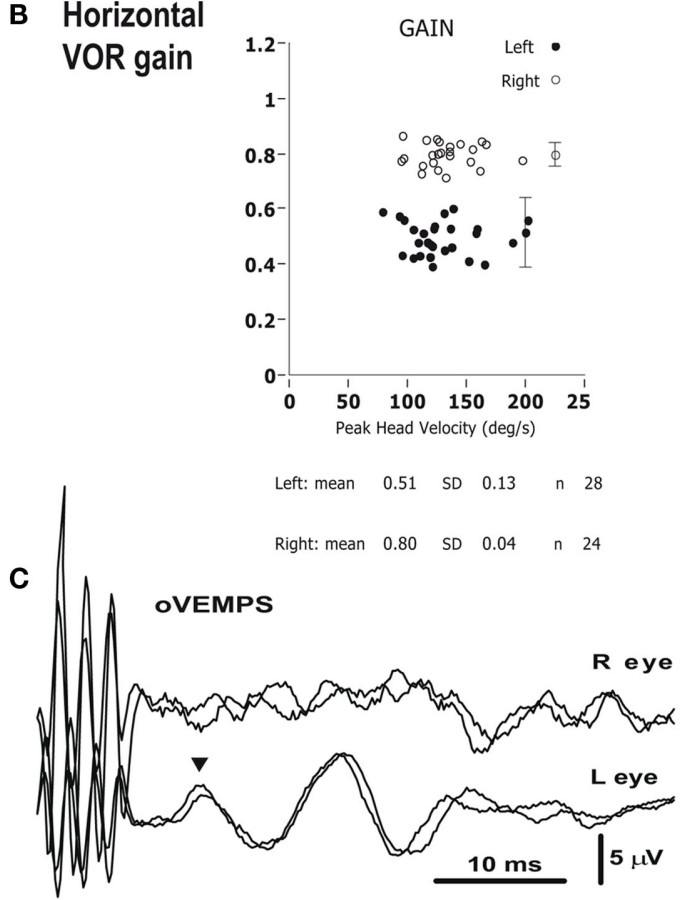

D cVEMPs
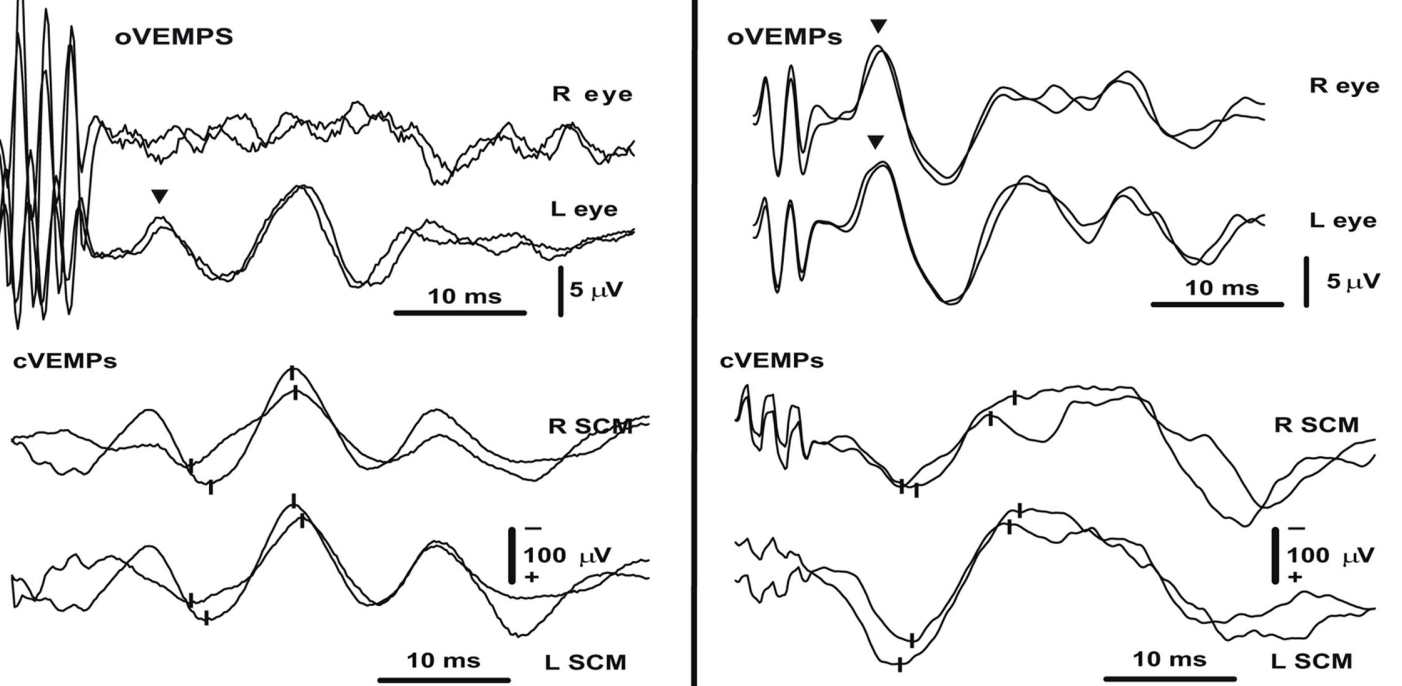

cVEMPS

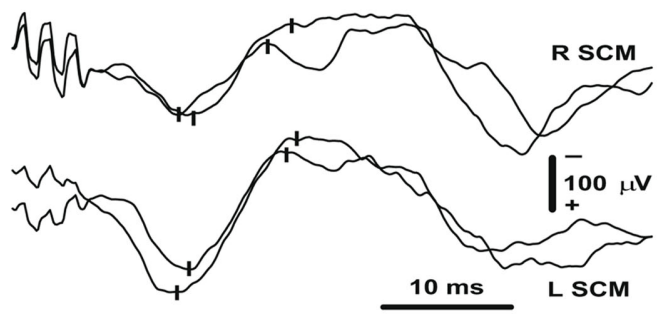

FIGURE 1 | Objective measures of vestibular function for the two testing sessions - acute phase (29.12.2010), and recovery phase (21.3.2011). (A) The figure shows many superimposed records of data from repeated head turns toward the affected (left side) and the healthy (right) side. Head velocity is red and the corresponding eye velocity is black. This is the vHIT test of horizontal canal dynamic function. The signs of head velocity for leftward impulses and of eye velocity for rightward impulses have been inverted for easier comparison. The eye velocity traces during the acute phase show a reduced gain for the left horizontal canal (as shown by reduced peak eye velocity in the panel for leftward impulses), and a large number of corrective saccades, mostly covert saccades since they occur during the head rotation,

confirming the inadequate left canal function. In the recovery phase, the gain in the left ear has increased to be within normal range. The calculated VOR gains (B) are shown and demonstrate the asymmetric VOR at attack and the return to symmetry 3 months later on 21 March. (C,D) Show the results of testing otolith function on the two occasions. (C) Tests of utricular function - averaged oVEMP responses to $500-\mathrm{Hz}$, 7-ms (1 ms rise-fall), short-duration tone burst BCV at Fz in the patient. The upper traces are for the right eye (caused by the affected left ear, since the oVEMP is a crossed response), and the lower traces for the left eye (caused by the healthy right ear). Two repeats are shown for each condition. The inverted triangles mark

(Continued) 


\section{FIGURE 1 | Continued}

the approximate times of the n10 potentials. During the acute phase the strong asymmetry in the amplitude of the $n 10$ response between left and right sides is evident, showing the absent dynamic utricular function on the left, with the response beneath the contralesional right eye being absent. By the time of the recovery phase there is a clear oVEMP n10 beneath the right eye showing the return of dynamic left utricular function so that the $\mathrm{n} 10$ amplitudes have become symmetrical just as normal healthy subjects show. (D) Tests of saccular function - cVEMPs to BCV recorded over both SCM muscles to the same $500 \mathrm{~Hz}$ Fz BCV stimulus on the two occasions. The upper traces are for the right sternocleidomastoid muscle (SCM), and the lower traces for the left SCM. The small vertical lines mark the $\mathrm{p} 13$ and $\mathrm{n} 23$ potentials. In contrast to the asymmetric oVEMP data in (C), during the acute phase the p13-n23 amplitudes on both sides are similar, and within the normal range, showing that the left saccular function is normal and so it appears that in this patient the saccular function was minimally affected by the neuritis. (Reprinted with permission from Laryngoscope, Manzari et al., 2011).
When studies with natural values of head accelerations (of the order of thousands of degrees/second/second) were used it became clear that the recovery of dynamic VOR for head rotations to the affected ear was small. The result is that for such rotations the eye velocity does not correct for head velocity, so that smear of the retinal image would occur during head turns. That result was demonstrated in animals as diverse as humans and guinea pigs (Gilchrist et al., 1998). The human data is instructive - in patients tested before and after UVL it was found that for ipsilesional rotations there was a large drop in gain immediately after the surgical loss and that over the next year for these same patients there was very little recovery in gain. The mean velocity gain at 1 week for 11 patients was $0.25 \pm 0.21(\mathrm{SD})$. The mean velocity gain at 1 year after UVL was $0.27 \pm 01.14$ (SD) (Halmagyi and Curthoys, 1988; Halmagyi et al., 1990). These patients are very unusual since tests before the UVL showed that their horizontal canal function was normal. They underwent the surgical UVL to treat intractable benign paroxysmal positioning vertigo which affected their posterior canal. Similar to horizontal canal function, the cVEMP and oVEMP measures of otolith function show that after UVL there is a permanent loss of contralateral oVEMP n10 and ipsilateral cVEMP p13-n23.

Is there any dynamic vestibular compensation at all? Yes, there does appear to be some improvement in VOR gain for ipsilesional rotations in the low frequency, low acceleration range. But it is not known how functionally effective this small gain recovery is. For higher head accelerations as encountered during most natural activities such as driving or sport, the evidence is that there is very little or no recovery of VOR gain.

The paradox is that despite this very inadequate recovery of slow phase eye velocity response to natural dynamic stimuli that many patients return to a normal lifestyle and are just not troubled by the UVL and the VOR loss that our precision measures show that they have. They have "compensated" but evidently not by synaptic changes in the vestibular nuclei since their measured eye velocity for unpredictable passive ipsilesional head rotations remains poor. Why then are they not troubled by their inadequate VOR? Two possibilities, which are not necessarily mutually exclusive are; (1) that the modest recovery of function for low frequency stimuli is enough or (2) that they are using other strategies to overcome their loss. Our preliminary evidence suggests the latter and that saccades may be the key. It has become apparent that it is the saccades which change over time. The great focus in the area of vestibular compensation has been on slow phase eye velocity without adequate recognition of the fact that changes in saccadic pattern are a very effective way of overcoming the loss (although see Berthoz,
1988). We suggest the focus for future studies of plasticity in the compensation of oculomotor responses in human patients after UVL should be on saccades, rather than slow phase eye velocity.

\section{SACCADES}

Our measures of responses to high acceleration head impulses shows that there are very distinctive patterns of saccadic performance in different patients and we think it is by adopting a particular saccadic strategy, even from the very earliest time after UVL that some patients recover so well. vHIT has shown us how very cleverly many patients with inadequate semicircular canal function can generate saccades which conceal their dysfunction. It now seems that these saccades may have a role in facilitating patient recovery.

Why are saccades so valuable for the patients? The very high velocity of the saccade will degrade the retinal image and in addition vision is suppressed during and around the time of a saccade (Richards, 1969; Matin, 1974). This saccadic suppression acts to eliminate the smear of the retinal image during inadequate slow phase eye velocity responses and it is this smear which is held by many to be one aversive component of vestibular loss. The saccade keeps the patient's gaze on the target, not by slow compensatory eye movements, but by a ballistic eye movement that eliminates the smeared retinal image which would have occurred during the head turn due to their inadequate VOR. So the saccade and saccadic suppression yield a visual stimulus, free of the retinal smear which otherwise would have occurred.

The contrast is shown well by comparing a UVL and a healthy subject when both are trying to maintain gaze during an active head movement. If one asks a patient with a UVL to maintain gaze on a spot on the wall while they actively, voluntarily turn their head abruptly to left or right, most patients and healthy subjects can quickly learn to do so. Precise high-speed measures show that a healthy subject maintains gaze by generating a smooth slow phase compensatory eye movement canceling the head movement. However the patient with a UVL maintains gaze for ipsilesional head turns by generating a small saccade at the onset of the head movement to correct their inadequate VOR during this active head movement (Black et al., 2005). This shows that during active head movements there is a substitution of a saccade for the deficient vestibular slow phase eye movement, much as postulated by Berthoz (1988). Once again such a saccade acts to minimize the effect of the UVL on the patients' permanent dynamic VOR deficit because the saccade minimizes retinal smear, and saccadic suppression further reduces the perceptual experience. More recently we have shown that some UVL patients 
can generate this saccade even during passive unpredictable, high acceleration head turns.

Our measures with scleral search coils (Weber et al., 2008) showed that some patients with a semicircular canal deficit on one side could manage to generate small corrective saccades actually during the unpredictable passive head movement to the affected side, so that at the end of the head turn to their affected side an overt saccades was not necessary to bring the eyes back on target. These small hidden saccades during the head rotation had concealed their inadequate VOR. We have called these hidden saccades covert saccades. It is important to realize that covert saccades can entirely obscure or conceal even a complete, total unilateral or bilateral loss of canal function. These covert saccades are very fast and they occur during the head rotation and they are almost impossible to detect by the naked eye, but are easily detected by and vHIT (MacDougall et al., 2009) and also by search coils (Weber et al., 2008, 2009).

These different patterns of saccadic responses lead to the hypothesis that the different groups of patients using these different strategies may show different patterns of vestibular compensation. During testing at the stage of the acute attack some VN patients already show changes in their pattern of saccades from overt to covert (see Figure 2). Looking back at the rehabilitation exercises of Cawthorne and Cooksey we think some of them are really optimized for (inadvertently) teaching people to generate this covert saccadic pattern.

One major question is what triggers covert saccades? The sensory input from the neck is one input which could possibly substitute for absent vestibular sensory input by virtue of the cervico-ocular reflex (COR) pathway from the neck to the vestibular nuclei. The slow phase eye velocity generated by the COR during passive turns is very small in healthy subjects but there is evidence for potentiation of this small COR after vestibular loss in monkeys with all six canals blocked (Yakushin et al., 2011). Similarly the gain of the COR is enhanced in some human patients with long term vestibular loss (reviewed in Yakushin et al., 2011). While this potentiation of the COR may be valuable for improving the slow phase eye responses to passive low acceleration head movements, the latency of the onset of the slow phase of the COR in human subjects during abrupt head impulses is so long (greater than $100 \mathrm{~ms}$ ) that it is not useful for generating slow compensatory eye movements to passive head turns with natural high accelerations (Halmagyi and Curthoys, 1987; Halmagyi et al., 1990). A patient with bilateral surgical vestibular loss for removal of neuromas had a good COR to low frequency sinusoidal rotations (rotating the body, while holding the head stationary) but could not generate slow phase eye velocity responses for about the first $100 \mathrm{~ms}$ of a head impulse. That result was confirmed in detail in another patient with bilateral surgical vestibular loss (Weber et al., 2009). However the COR may have a role in triggering covert saccades. The 2009 patient with total bilateral surgical loss was tested in the head impulse paradigm (Weber et al., 2009) and although they had no compensatory slow phase eye velocity during the first $100 \mathrm{~ms}$ they showed excellent performance in generating covert saccades to over 300 head impulses with randomized directions. This patient's covert saccades were always in the correct direction with an average latency of about $124 \mathrm{~ms}$ from the onset of the head movement. The excellent performance of this patient with totally absent bilateral vestibular function shows that covert saccades cannot be due to vestibular input, but whatever is triggering them is providing a highly reliable signal. It is possible that the sensory input from the neck at the onset of the head turn may be the trigger stimulus for generating a covert saccade. It is even possible that the potentiated COR enhances oculomotor responses to low frequency stimuli and serves to trigger covert saccadic responses for high acceleration head rotations.

\section{VESTIBULAR COMPENSATION AND REHABILITATION}

These new measurement techniques have given us a new insight into just how well patients can recover. A patient of our colleague, Dr. Leonardo Manzari, of Cassino, Italy leads a perfectly normal lifestyle. But as a young boy, over 50 years ago, he received the ototoxic antibiotic streptomycin, systemically to treat an infection. Our recent objective tests showed that, almost certainly because of the streptomycin destroying the hair cells in the vestibular sense organs of his inner ears at a very young age, his peripheral vestibular functions is totally and completely absent, bilaterally absent vHIT, absent oVEMPs, absent cVEMPs. However his life
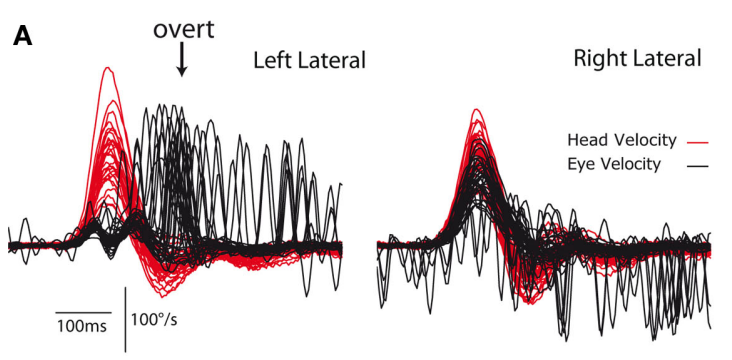

FIGURE 2 | Video head impulse test data from a patient with a left unilateral vestibular neuritis 2 days after the attack shows spontaneous nystagmus, and very low VOR gain for ipsilesional (leftward) head turns from the affected side (A). Head velocity is red, eye velocity is black. The eye velocity has been inverted to allow comparison of the head velocity stimulus and the eye velocity response. For rotations to the patient's healthy (right) side the eye velocity fairly closely matches head velocity. For rotations to the

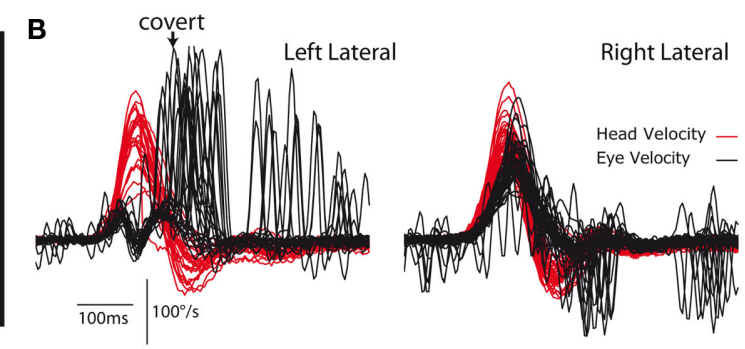

affected (left) side the eye velocity is substantially smaller than head velocity. The saccades occur at or after the end of the head rotation for the first occasion and so are classed as overt saccades (A), whereas at testing this patient just hours later the saccades tend to occur earlier, during the head rotation. So at the second test (B) only an hour later than (A) the subject's compensatory saccades are already starting to separate into separate groups of covert and overt populations (right plots). 
is completely normal - he drives, he plays sport. He is untroubled by any kind of postural instability, even when tested in darkness. There is no evidence of ataxia. He generates covert saccades which are almost invisible to the naked eye but which are clearly shown with vHIT measures. It is quite by chance that these facts were discovered - he visited the clinic because of auditory sensations due to dehiscence of his labyrinth and was given the full vestibular test battery which showed the absence of vestibular function. Of course he has had 50 years to learn these behaviors but the point is that the behaviors are completely successful. But it is only by using the new tests of canal and otolith function that we know that he has absent canal and otolith function. His performance is the benchmark of stability which can be achieved without vestibular function.

\section{SUMMARY}

The vestibular system is a very fundamental system whose activity affects many other motor systems, so disruption of vestibular function has far-reaching consequences. Some symptoms recover, in the sense that vestibular-dependent performance resumes, some do not. Over time the patient resumes their lifestyle because of this patchwork recovery and because they learn a variety of new behaviors to allow normal function. However if the appropriate tests are carried out probing vestibular function and preventing some of the other "tricks" then the permanent loss of vestibular function is very clear.

\section{REFERENCES}

Berthoz, A. (1988). The role of gaze in compensation of vestibular disfunction: the gaze substitution hypothesis. Prog. Brain Res. 76, 411-420.

Black, R. A., Halmagyi, G. M., Thurtell, M. J., Todd, M. J., and Curthoys, I. S. (2005). The active head-impulse test in unilateral peripheral vestibulopathy. Arch. Neurol. 62, 290-293.

Colebatch, J. G., and Halmagyi, G. M. (1992). Vestibular evoked potentials in human neck muscles before and after unilateral vestibular deafferentation. Neurology 42, 1635-1636.

Colebatch, J. G., Halmagyi, G. M., and Skuse, N. F. (1994). Myogenic potentials generated by a click-evoked vestibulocollic reflex. J. Neurol. Neurosurg. Psychiatr. 57, 190-197.

Curthoys, I. S. (2010). A critical review of the neurophysiological evidence underlying clinical vestibular testing using sound, vibration and galvanic stimuli. Clin. Neurophysiol. 121, 132-144.

Curthoys, I. S., and Halmagyi, G. M. (1995). Vestibular compensation: a review of the oculomotor, neural, and clinical consequences of unilateral vestibular loss. J. Vestib. Res. 5, 67-107.

Curthoys, I. S., and Halmagyi, G. M. (1999). Vestibular compensation. Adv. Otorhinolaryngol. 55, 82-110.
Curthoys, I. S., Kim, J., McPhedran, S. K., and Camp, A. J. (2006). Bone conducted vibration selectively activates irregular primary otolithic Exp. Brain Res. 175, 256-267.

Curthoys, I. S., and Vulovic, V. (2011). Vestibular primary afferent the guinea pig. Exp. Brain Res. 210, 347-352.

Gilchrist, D. P., Curthoys, I. S., Cartwright, A. D., Burgess, A. M., Topple, A. N., and Halmagyi, M. (1998). High acceleration impulsive rotations reveal severe longterm deficits of the horizontal vestibulo-ocular reflex in the guinea pig. Exp. Brain Res. 123, 242-254.

Halmagyi, G. M., and Curthoys, I. S. (1987). "Human compensatory slow eye movements in the absence of vestibular function," in The Vestibular System: Neurophysiologic and Clinical Research, eds M. D. Graham and J. L. Kemink (New York: Raven Press), 471-479.

Halmagyi, G. M., and Curthoys, I. S. (1988). A clinical sign of canal paresis. Arch. Neurol. 45, 737-739.

Halmagyi, G. M., Curthoys, I. S., Cremer, P. D., Henderson, C. J., Todd, M. J., Staples, M. J., and D'Cruz, D. M. (1990). The human horizontal vestibulo-ocular reflex in vestibular neurons in the guinea pig. responses to sound and vibration in

Taking such patients into a clinic and trying to assess the mechanism of their recovery, based on a few classical measures of oculomotor performance using unnaturally low values of head acceleration, may not reveal the subtle but effective compensatory strategies which people use in real-life. It was only by using highspeed, high resolution measures of eye movement that we detected the very small covert saccades during the passive high acceleration head movements which may hold the key to understanding compensation of oculomotor symptoms. We think saccades are the new frontier in vestibular compensation - and the major question now is what triggers them.

Substitution of other responses can effectively conceal the vestibular deficit and so protect the patient from receiving smeared retinal images during head movements. The permanent deficit of dynamic VOR function to natural accelerations after UVL shows that vestibular rehabilitation of UVL patients should be aimed at developing new saccadic behavior which substitutes for the dynamic vestibular loss, rather than trying to restore something which cannot be restored.

\section{ACKNOWLEDGMENTS}

This work was supported by a grant from the Garnett Passe and Rodney Williams Memorial Foundation to Dr. Hamish MacDougall.

response to high-acceleration stimulation before and after unilateral vestibular neurectomy. Exp. Brain Res. 81, 479-490.

Halmagyi, G. M., Weber, K. P., and Curthoys, I. S. (2010). Vestibular function after acute vestibular neuritis. Restor. Neurol. Neurosci. 28, 37-46.

Iwasaki, S., McGarvie, L. A., Halmagyi, G. M., Burgess, A. M., Kim, J., Colebatch, J. G., and Curthoys, I. S. (2007). Head taps evoke a crossed vestibulo-ocular reflex. Neurology 68, 1227-1229.

MacDougall, H. G., Weber, K. P., McGarvie, L. A., Halmagyi, G. M., and Curthoys, I. S. (2009). The video head impulse test: diagnostic accuracy in peripheral vestibulopathy. Neurology 73, 1134-1141.

Manzari, L., Burgess, A. M., MacDougall, H. G., and Curthoys, I. S. (2011). Objective verification of full recovery of dynamic vestibular function after superior vestibular neuritis. Laryngoscope 121, 2496-2500.

Matin, E. (1974). Saccadic suppressionreview and an analysis. Psychol. Bull. 81, 899-917.

Murofushi, T., Curthoys, I. S., Topple, A. N., Colebatch, J. G., and Halmagyi, G. M. (1995). Responses of guinea pig primary vestibular neurons to clicks. Exp. Brain Res. 103, 174-178.
Richards, W. (1969). Saccadic suppression. J. Opt. Soc. Am. 59, 617-623.

Rosengren, S. M., Todd, N. P. M., and Colebatch, J. G. (2005). Vestibularevoked extraocular potentials produced by stimulation with boneconducted sound. Clin. Neurophysiol. 116, 1938-1948.

Rosengren, S. M., Welgampola, M. S., and Colebatch, J. G. (2010). Vestibular evoked myogenic potentials: past, present and future. Clin. Neurophysiol. 121, 636-651.

Uchino, Y., Sasaki, M., Sato, H., Bai, R., and Kawamoto, E. (2005). Otolith and canal integration on single vestibular neurons in cats. Exp. Brain Res. 164, 271-285.

Weber, K. P., Aw, S. T., Todd, M. J., McGarvie, L. A., Curthoys, I. S., and Halmagyi, G. M. (2008). Head impulse test in unilateral vestibular loss: vestibulo-ocular reflex and catch-up saccades. Neurology 70, 454-463.

Weber, K. P., Aw, S. T., Todd, M. J., McGarvie, L. A., Curthoys, I. S., and Halmagyi, G. M. (2009). Horizontal head impulse test detects gentamicin vestibulotoxicity. Neurology 72, 1417-1424.

Yakushin, S. B., Kolesnikova, O. V., Cohen, B., Ogorodnikov, D. A., Suzuki, J.-I., Della Santina, C. C., Minor, L. B., and Raphan, T. (2011). Complementary gain modifications 
of the cervico-ocular (COR) and angular vestibulo-ocular (aVOR) reflexes after canal plugging. Exp. Brain Res. 210, 549-560.

Conflict of Interest Statement: The authors declare that the research was conducted in the absence of any commercial or financial relationships that could be construed as a potential conflict of interest.

Received: 07 November 2011; paper pending published: 30 November 2011; accepted: 03 February 2012; published online: 28 February 2012.
Citation: MacDougall HG and Curthoys IS (2012) Plasticity during vestibular compensation: the role of saccades. Front. Neur. 3:21. doi: 10.3389/fneur.2012.00021

This article was submitted to Frontiers in Neuro-otology, a specialty of Frontiers in Neurology.
Copyright (c) 2012 MacDougall and Curthoys. This is an open-access article distributed under the terms of the Creative Commons Attribution Non Commercial License, which permits noncommercial use, distribution, and reproduction in other forums, provided the original authors and source are credited. 